Husar, Peter; Berkes, Sebastian; Götze, Andreas; Henning, Günter; Plagwitz, KaiUwe:

\title{
Adaptive SNR-Anhebung von VEP mit Statistik höherer Ordnung
}

\author{
Zuerst erschienen in: Biomedizinische Technik = Biomedical Engineering. - Berlin [u.a.] : \\ de Gruyter. - 48 (2003), S1, S. 180-181. \\ Jahrestagung der Deutschen, der Österreichischen und der \\ Schweizerischen Gesellschaften für Biomedizinische Technik ; \\ (Salzburg) : 2003.09.25-27 \\ Erstveröffentlichung: 2003 \\ Datum Digitalisierung: $2009-10-23$ \\ ISSN (online): $\quad$ 1862-278X \\ ISSN(print) 0013-5585 \\ DOI: $\quad$ 10.1515/bmte.2003.48.s1.180 \\ [Zuletzt gesehen: 2019-12-12]
}

„Im Rahmen der hochschulweiten Open-Access-Strategie für die Zweitveröffentlichung identifiziert durch die Universitätsbibliothek IImenau."

"Within the academic Open Access Strategy identified for deposition by IImenau University Library."

„Dieser Beitrag ist mit Zustimmung des Rechteinhabers aufgrund einer (DFGgeförderten) Allianz- bzw. Nationallizenz frei zugänglich.“

"This publication is with permission of the rights owner freely accessible due to an Alliance licence and a national licence (funded by the DFG, German

Research Foundation) respectively."

\section{DFG}

Nationallizenzen 


\title{
ADAP'TIVE SNR-ANHEBUNG VON VEP MIT S'IATISTIK HÖIIERER ORDNUNG
}

\author{
P. Ilusar', S. Berkes' ${ }^{2}$, A. Götze', (i.l Henning', K.-U. Plagwitz' \\ 'Institut Biomedizinische Technik und Informatik, TU IImenau, Deutschland \\ 'Lildith GmbH, Ilmenau, Deutschland \\ peter.husar(a)tu-ilmenau.de
}

\section{SI MIMIARI}

listi.l evoked polentials (VEP) are diagnostic features III asisisisment of the visual system. Because of very low SiVK (Signal-10-Noise Ratio) repetitive stimulus responses are alveraged to obtain sufficient signal strength. successile aleraging is time consumptive. In multichannel HEG recordings we save the measurement time Impros ing the SNR simultaneously by adaptive channel delity control. The adaptive algorithm is controlled by higher-order correlation coefficient, which is a scalar gir ing the similarity of multi-channel recordings.

\section{I.I.T.FITUNG}

Iisuell evozierte Potentiale (VEP) sind objektive Funkunnsindikatoren des visuellen Systems. Da sie sehr schwich sind, werden sie durch reizsynchrone Mittelung herausgerechnet. Dies führt zu langen und inakzeptablen Messzeiten. In diesem Beitrag wird eine Methode Lur schnellen SNR-Anhebung (Signal-to-Noise-Ratio) rorgestellt. Eine Sensoranordnung zur EEG/MEGErfassung kann als nachrichtentechnische Sensormatrix interpretiert werden. Ansätze zur SNR-Verbesserung der evozierten kortikalen Aktivität mit Hilfe räumlicher Filter sınd bekannt. Bei der Analyse sensorischer Systeme, bei den die kortikalen Strukturen bekannt sind, ist von eınem räumlichen Filter allein kein Beitrag zu erwarten. Ausgehend davon, dass Reizantworten sich langsain ausbreiten $(1 \ldots 10 \mathrm{~m} / \mathrm{s})$ und an den Sensoren zeitverschoben auftreten, eignen sich zur Verbesserung des SNR spatiotemporale Filter. Die Voraussetzungen zur Anwendung des spatiotemporalen Beamforming sind nicht erfüllt, so dass praktikable Methoden gefragt werden.

\section{METHODE}

Gehen wir von einem additiven Signal-Rausch-Modell $x(t)=A_{i} s\left(t+\tau_{i}\right)+n_{i}(t) \quad \begin{aligned} & \text { zeitverschobener Sig- } \\ & \text { nale aus, wobei } A_{i} \text { die }\end{aligned}$ Amplitude des Signals $s, \tau_{i}$ die Verzögerung und $n_{i}$ normalverteiltes $N \sim(0 ; 1)$ weißes Rauschen im $i$-ten Kanal sind. Das Rauschen ist räumlich und zeitlich unkorreliert. Durch die En$y(t)=\frac{1}{N} \sum_{i=1}^{N} x_{i}(t) \begin{aligned} & \text { le wird die Rauschleistung um } \\ & \text { die Mittelungsordnung } N \text { redu- }\end{aligned}$ ziert. Die Mitlelung verursacht wegen des Phasenjitters Dämpfung des Signals um den Faktor

$$
D=\frac{1}{N} \mid \sum_{i=1}^{N} \exp \left(-j \omega \tau_{i}\right) \text {. Insgesamt führt die En- }
$$

semblemittelung zur Veränderung des ursprünglichen $S N R$ um den Faktor $S=D . N^{\prime 1.5}$. Für den Idealfall $D=1$ müssen die Kanalverzögerungen identisch bzw. deren Laufzeitdifferenzen Null sein. Dies ist machbar mit zusätzlichen steuerbaren Delays. Da die Signalform a priori nicht bekannt ist und auch kein Referenzsignal vorliegt, müssen hierfür modellfreie Ansätze verwendet werden [1]. Zur Schätzung der Zeitverschiebung von qualitativ gleichen aber unbekannten Signalen eignet sich die Kreuzkorrelation. Mit herkömmlichen Statistiken zweiter Ordnung wären $N^{2}$ Korrelationskoeffizienten auszuwerten. Das würde den Adaptionsalgorithmus

$$
R=\frac{\sum_{k=1}^{M} \prod_{i=1}^{N} x_{i}(k)}{\prod_{i=1}^{N}\left(\sum_{k=1}^{M} x_{i}^{N}(k)\right)^{\frac{1}{N}}}
$$
umständlich und unüberschaubar machen. Hierzu eignet sich besser der multidimensionale Korrelationskoeffizient (HOC) nach [2]. Dieser ergibt einen Skalarwert zwischen -1 und +1 und gibt den Grad der Ähnlichkeit der Kanäle an (für den Korrelationskoeffizien$\tau_{i}(n+1)=\tau_{i}(n)+\mu \nabla_{i}^{N}(n) \quad \begin{aligned} & \text { ten nach Pearson } \\ & N=2) \text {. Der HOC }\end{aligned}$ $\nabla_{i}(n)=\frac{\partial R(n)}{\partial \tau_{i}} \approx \frac{\Delta R(n)}{\Delta \tau_{i}} \quad \begin{aligned} & \text { wird zur Schätzung } \\ & \text { des Gradienten für } \\ & \text { den Rekursionsalgo- }\end{aligned}$ rithmus verwendet.

\section{ERGEBNISSE}

Die vorgeschlagene Methode wurde mit simulierten Daten entsprechend dem additiven Signalmodell getestet. Abb.1 zeigt eine 16-kanalige Aufnahme eines künstlichen VEP im Rauschen. Nach etwa 30 Rekursionssschritten ist die Adaption abgeschlossen (Abb. 2), dabei wird ein HOC von 0.7 und eine Anhebung des SNR um 3 dB (Abb.3) erreicht. Reale Daten wurden bei transienter kleinflächiger zentraler Lichtstimulation 16kanalig über dem visuellen Kortex aufgenommen und über 64 Einzelantworten gemittelt. Der Algorithmus zeigt für reale Daten (Abb.4) qualitativ einen ähnlichen Verlauf und bestätigt den Lösungsansatz. 


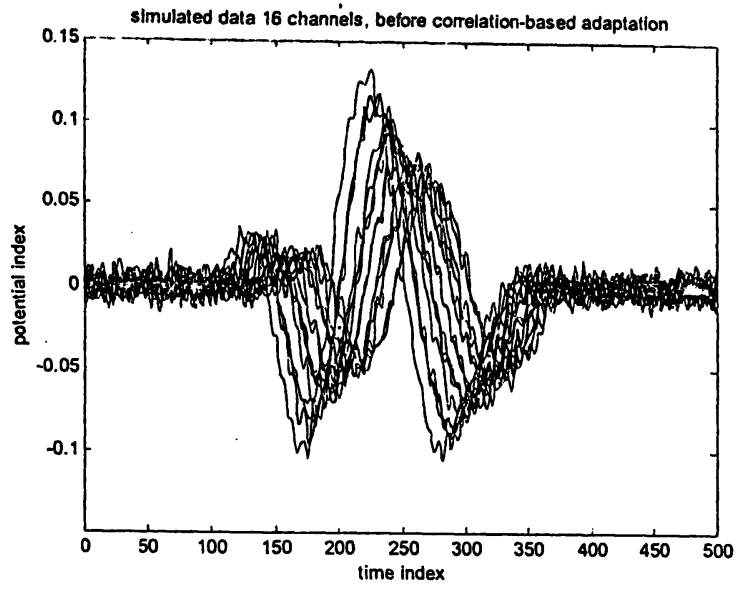

Abb.1: Simulierte Reizantworten im Rauschen $(\mathrm{N}=16)$.

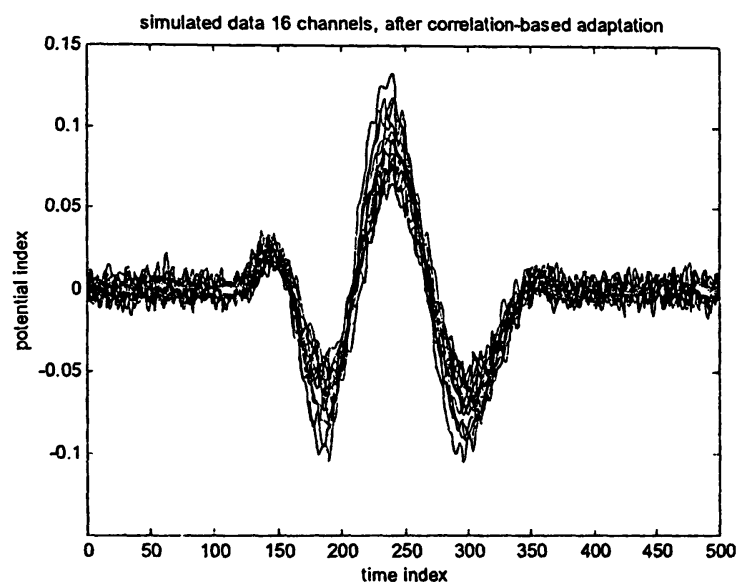

Abb.2: Abgeschlossene Adaption aus Abb.1

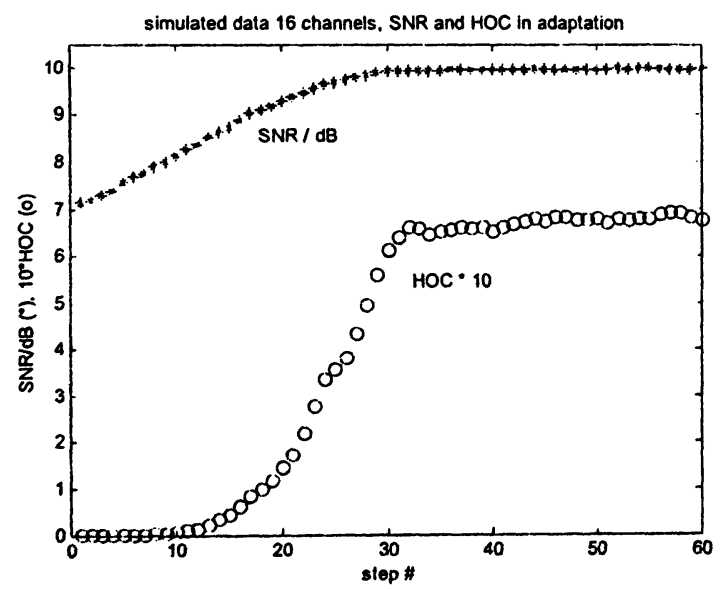

Abb.3: SNR (*) und HOC (o) während der Adaption

\section{DISKUSSION}

Die erreichbare Anhebung des SNR ergibt sich maßgeblich aus der Minimierung des Phasenjitters zwischen den Kanälen, so dass sie weder bei sımulierten noch bei realen Daten im voraus quantifiziert werden kann. Daher ist auch nicht überraschend, dass in diesem Fall bei realen Daten eine höhere Anhebung als bei simulierten Daten erreicht werden konnte. Bei realen Daten wirkt sich weiterhin eine Reduktion der immer vorhandenen räumlichen Kreuzkorrelation positiv aus. Bei größeren Laufzeitdifferenzen bzw. einer Phasenverschiebung von mehr als $\pi$ kann das globale HOC-Maximum verfehlt werden. Diese Gefahr steigt, wenn sich die qualitativen Verläufe in den Kanälen wesentlich unterscheiden.

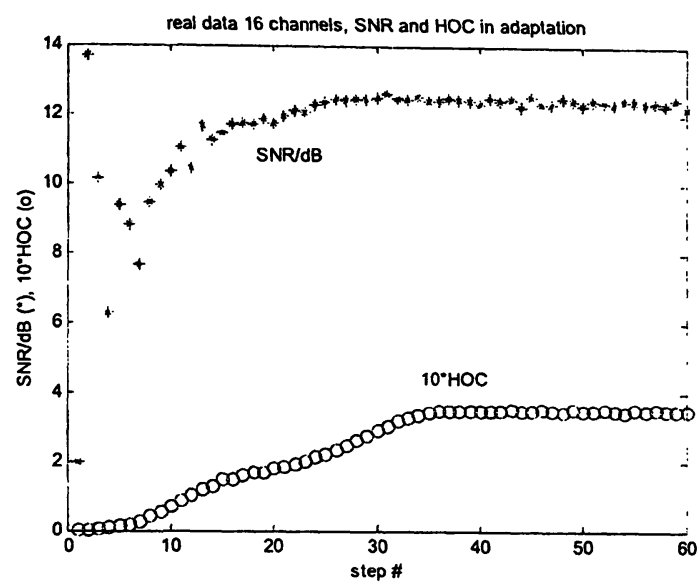

Abb.4: SNR (*) und HOC (o) bei realen Daten

\section{SCHLUSSFOLGERUNGEN}

Ein adaptives Kanaldelay, das mit Hilfe der Korrelation höherer Ordnung auf maximale Ähnlichkeit zwischen den Kanälen optimiert wird, kann zur Erhöhung des SNR beitragen. Die erreichbare Anhebung des SNR ist von dem Anfangsjitter und der räumlichen und zeitlichen Korrelation des Rauschens maßgeblich abhängig. Bei mehrphasigen Signalen kann nicht gewährleistet werden, dass das globale SNR-Maximum erreicht wird. Mit zunehmendem Phasenjitter relativ zur Periode des mehrphasigen Signals nimmt die Gefahr zu, dass sich der Algorithmus in ein lokales Optimum verläuft.

\section{DANKSAGUNG}

Diese Arbeit wurde im Forschungsprojekt „Neue Methoden der Optik und der Biosignalverarbeitung für die ophthalmologische Funktionsdiagnostik" durch dis Thüringer Ministerium für Wissenschaft, Forschung und Kunst (B 309 - 00016) gefördert.

\section{LITERATUR}

[1] Husar, P., Berkes, S., Henning. (i., Schcllhorn. K.. Schlegelmilch, F.: Beaming Signal Sources it! Measurement of Focal Visual livoked corrticial l'otentials, 23rd Aun. Intntl. Cont. IFEE LEMBSS, 2(1)1, Istanbul, Turkey, cont. proc., paper nr 100s, pp.1.t

[2] Henning, (i., Husir. P.: Statistical Detecluen of

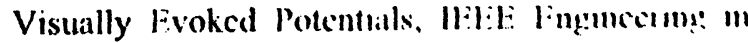
Medicine and Biology Vol.14(1995). 4. PN. 3iso . :911 